\title{
Generalized Trends in Atmospheric Attenuation and The Importance of Accurate Extinction Calculation
}

\author{
Agam Gupta ${ }^{1}$ and Reema Gupta ${ }^{1}$
}

${ }^{1}$ Ardrey Kell High School, Charlotte, NC, USA

\section{ABSTRACT}

The purpose of this literature review is to explore and quantify the causes of attenuation of electromagnetic radiation, as well as to develop generalized trends for extinction as a function of influential factors (wavelength of incident electromagnetic wave, altitude of observatory, variations in seasons). This literature review also discusses the implications of precise measurement of atmospheric attenuation in determining intrinsic luminosity of stars in astronomical photometry and significance of accurate measurement for atmospheric extinction to precisely calculate the mass, size and lifetime of stars in the main sequence of the Hertzsprung-Russell plot diagram.

\section{Introduction}

Atmospheric attenuation is the reduction of intensity of electromagnetic radiation after interaction with suspended atmospheric particles. The precise measurement of atmospheric extinction is a major factor and has many implications such as in satellite communications, radio broadcasting, television, telephone communication. Furthermore, accurate measurement of atmospheric attenuation is critical for good quality ground- based Astronomical Photometry. BougerBeer- Lambert's law establishes the relationship between expected intensity extinction due to the atmosphere for monochromatic light waves (Capua et. al, 2014; Ballentyne and Lovett, 1970, pg.188; Baker et. al, 2014). This relationship is given by:

$$
I=I_{0} * e^{\beta^{*} X}
$$

wherein $\boldsymbol{I}$ is the intensity of monochromatic light after passing the atmosphere, $\boldsymbol{I}_{\boldsymbol{o}}$ is the intensity flux of light before entering the atmosphere, $\boldsymbol{\beta}$ is the total extinction (in magnitudes/ air mass) coefficient and $\boldsymbol{X}$ is the number of air masses (elaborated upon below). The total extinction coefficient $\boldsymbol{\beta}$ can be calculated by:

$$
\boldsymbol{\beta}=\boldsymbol{\beta}_{\text {mol }}+\boldsymbol{\beta}_{\text {ray }}+\boldsymbol{\beta}_{\text {aer }}
$$

where $\boldsymbol{\beta}_{\text {mol }}$ is the extinction coefficient of molar absorption, $\boldsymbol{\beta}_{\text {ray }}$ is the extinction coefficient of rayleigh scattering and $\boldsymbol{\beta}_{\text {aer }}$ is the extinction coefficient of aerosol scattering, phenomenon discussed below.

The Bouger's law fundamentally assumes the following conditions:

- The isotropy and homogeneity of the atmospheric particles.

- The incident light has to be monochromatic.

- The absorbers/ scatterers act independently of each other. 
- The incident influx doesn't influence particles.

- The incident radiation is in the form of parallel rays, each travelling the same distance in the influential atmosphere.

This review comprises of quantitative and qualitative elaboration upon air mass, the 3 primary components (Molecular Absorption, Rayleigh Scattering, Aerosol Scattering) of attenuation, and synthesizes existing literature to develop generalized trends as a function of altitude of observatory, wavelength of incident light, and seasonal variations. Furthermore, the paper exclusively discusses the importance of accurate measurement of extinction for Atmospheric Photometry and precise calculations of stellar characteristics.

The paper does not include all applications and implications of accurate measurements of attenuation. Rather, its importance in astronomical photometry and determining stellar characteristics is discussed exclusively. The paper does not signify the importance of interstellar extinction, which astronomers should take into account in calculating the intrinsic luminosity and subsequently determining stellar characteristics.

\section{Literature Review}

\section{Air Mass}

Air mass $(\boldsymbol{X})$ is defined as a body of air with uniform humidity, pressure and temperature. 1 air mass is considered the amount of air one's looking through while viewing the zenith. The amount of air mass, or the amount of atmosphere source light has to travel across varies depending upon the extinction material, refraction effects and variation of air density. For small zenith angles, $\boldsymbol{X}=\sec (k)$ is a reasonable approximation. For extreme zenithal distances, Hardie (1962) noted that $\boldsymbol{X}$ can be determined by:

$$
\boldsymbol{X}=\sec (k)-0.0018167(\sec (k)-1)-0.002875(\sec (k)-1)^{2}-0.0008083(\sec (k)-1)^{3}
$$

There are several other equations, including that of Young and Krasten(1989) which states $\boldsymbol{X}=(\cos (k)+$ $\left.0.50572(6.07995+90-k)^{-.1 .6364}\right)^{-1}$ and that of Young and Irvine $(1967)$ which states that $X=\sec (k)(1-$ $\left.0.0012\left(\sec ^{2} k-1\right)\right)$. Though there are minute differences in the equations of calculation of air mass due to zenith angle, air density and refraction effects, a general overall representation of air mass can be given by Rozenberg's (1966) equation:

$$
X=\left(\cos (k)+0.025 e^{(-11 \cos (k))}\right)^{-1}
$$

where $\boldsymbol{X}$ is the air mass and $\boldsymbol{k}$ is the zenith angle, in degrees. It is evident from the equation that as the celestial object's angular distance from the zenith increases, air mass tends to increase. It is also true that atmospheric extinction tends to increase as the angle increases.

\section{Coefficient of Atmospheric Extinction}

Atmospheric extinction is caused by 3 primary sources: 


\section{Molecular absorption}

Molecular absorption is the conversion of radiant energy into oscillation energy of molecules and elements dispersed in the atmosphere including ozone, $\mathrm{CO}_{2}$ and water vapor that are mostly suspended in the stratosphere, between 10 to $35 \mathrm{~km}$. For electromagnetic waves in the visible spectrum, molecular absorption is largely negligible in the range $0<$ $\boldsymbol{k}<75$. At sea level, the extinction due to molecular absorption $\left(\boldsymbol{\beta}_{\mathrm{mol}}\right)$ is around 0.016 magnitude per air mass (Schaeffer, B.E as cited in Green, 1992).

\section{Rayleigh Scattering}

Rayleigh Scattering is the scattering of photons due to electromagnetic interactions between source light and molecules suspended in the air like Nitrogen $\left(\mathrm{N}_{2}\right)$ and Oxygen $\left(\mathrm{O}_{2}\right)$. Rayleigh Scattering applies to cases wherein scattering particles are very small $(\boldsymbol{d}<<\lambda)$. The intensity after attenuation is given by the equation:

$$
I=I_{o} * \frac{\left(1+\cos ^{2}(\boldsymbol{\theta})\right)}{2 R^{2}} *\left(\frac{2 \pi}{\chi}\right)^{4} *\left(\frac{n^{2}-1}{n^{2}+2}\right)^{2} *\left(\frac{d}{2}\right)^{6}
$$

where $\boldsymbol{I}$ is the intensity of light scattered, $\boldsymbol{I}_{\boldsymbol{o}}$ is the incident light intensity, $\boldsymbol{R}$ is the distance between the particle and the observer, $\boldsymbol{\theta}$ is the scattering angle, $\boldsymbol{n}$ is the refractive index and $\boldsymbol{d}$ is the effective diameter of the particle, and $\boldsymbol{\lambda}$ is the wavelength of incident light. This equation holds true for particles whose size is less than $10 \%$ of that of the wavelength of the light being scattered.

It is evident from this that the intensity, or the amount of light scattered, is inversely proportional to the wavelength of the electromagnetic wave. Thus, in the visible spectrum, blue- violet light, with the smallest wavelength, is more probable to scatter than red when the sun is on the zenith.

An approximation for the $\boldsymbol{\beta}_{\text {ray }}$ was given by Hayes and Latham(1975) and Penndorf(1957) as a function of wavelength of light and height of observatory:

$$
\boldsymbol{\beta}_{\text {ray }}=9.4977 * 10^{-3} * \chi^{-4} * e^{\left(\frac{-h}{0.776}\right)} *\left[\frac{(n-1) \chi}{(n-1) \chi}\right]^{2}
$$

Here, $\boldsymbol{h}$ equals the height of the observatory and $\left[\frac{(n-1) \chi}{(n-1)]^{2}}\right]^{2}$ is the index of refraction term.

\section{Aerosol scattering}

Aerosol scattering is due to particles suspended in the atmosphere including dust, water droplets, clouds, artificial pollutants, smoke and volcanic soot. Aerosol scattering greatly fluctuates from time to time due to variations in aerosol components (Schaefer, 1992; Hayes and Latham, 1975; Green, 1992; Vargas et. al; 2002). At sea level, the average aerosol scattering coefficient $\left(\boldsymbol{\beta}_{\text {aer }}\right)$ is about 0.12 magnitude per air mass (Green, 1992). The formula for Aerosol scattering encompasses the use of Mie theory, which is used if the particles' diameter are within $10 \%-110 \%$ of the wavelength of the incident radiation.

The Mie Theory, as expressed and derived by McLinden (1999), shows that the aerosol extinction cross- section coefficient $(\boldsymbol{\sigma})$ is equal to:

$$
\boldsymbol{\sigma}=\frac{2 \pi}{k^{2}} \sum_{n=1}^{\infty} \mathbf{\square}(2 n+1) \operatorname{Re}\left(a_{n}+b_{n}\right)
$$


and the aerosol extinction coefficient $\boldsymbol{\beta}_{\text {aer }}=N^{*} \boldsymbol{\sigma}$ where $k=2 \pi / a, a$ is the particle radii, $\boldsymbol{N}$ is the number of particles suspended in the atmosphere, $R e$ is the real part of the sum of complex numbers, $\boldsymbol{a}_{\boldsymbol{n}}$ and $\boldsymbol{b}_{\boldsymbol{n}}$ are coefficients calculated by synthesizing spherical bessel functions of the first order.

\section{Generalized Trends}

The total extinction coefficient $(\boldsymbol{\beta})$ fluctuates as a factor of the altitude of the observing site, the concentration, size and inherent chemical nature of particles present in the atmosphere, the wavelength of incident influx, and the season in which observations are made. These factors are taken into consideration in the calculation of the magnitude of aerosol and rayleigh scattering, but the general trends are as follows:

\section{Altitude of Observatory}

As the altitude of the observatory increases, the average atmospheric extinction tends to decrease. Green (1992) found out that as the height of observation increases from $0 \mathrm{~km}$ to $3 \mathrm{~km}$ above sea level, the average coefficient of atmospheric extinction decreases by $50 \%$ or more for all zenith distances. Siher, Benkhaldoun and Fossat (2003) conducted a comprehensive diurnal study on six different observatories in Culgoora, Australia; Kumbel, Uzbekistan; Oukaimeden, Morocco; Izana, Spain; La Silla, Chile; Stanford, United States from data collected from 1989- 1997 from the IRIS databases. The data concluded that the atmosphere extinction coefficient of higher sites - Oukaimeden (2700m), Izana (2395m), Kumbel (2300m), La Silla (2400m) - was considerably lower than that of Culgoora, which is situated 200m over sea level.

\section{Wavelength of Incident Electromagnetic Light}

The wavelength of the incident radiation flux influences the amount of attenuation. Generally, monochromatic light of shorter wavelengths have a shorter atmospheric extinction coefficient and are attenuated less than monochromatic light of longer wavelengths. The wavelength of the incident light is factored into the formulas of Rayleigh Scattering and Aerosol Scattering.

Hayes and Latham (1975), in their extensive study to improve past data of atmospheric extinction at Lick Observatory and Palomar Mountain (California) and Cerro Tololo (Chile), provide significant data which shows the relationship between wavelength of incident light and the atmospheric extinction coefficient. Hayes and Latham (1975) used monochromatic light radiation of different wavelengths (ranging from $3450 \AA$ to $5000 \AA$ for Lick and $3400 \AA$ to $5000 \AA$ for Cerro Tololo) to fabricate a theoretical extinction coefficient and then observe the data to calculate the total atmospheric extinction coefficient. In the data corresponding to the Lick observatory, the extinction coefficient was observed to be 0.708 for $3450 \AA$ and 0.207 for $5000 \AA$. Similarly, the observed data at Cerro Tololo computed the extinction coefficient to be 0.680 for $3450 \AA$ and 0.179 for $5000 \AA$. Vargas, Benitez, Bajo and Vivas (2002) in their study to determine the atmospheric extinction coefficient at the Physics Department Astronomical Observatory of the University of Extremadura in Spain utilized electromagnetic waves corresponding to filters B, V, R, I which have wavelengths equal to $445 \mathrm{~nm}, 551 \mathrm{~nm}, 658 \mathrm{~nm}$ and $806 \mathrm{~nm}$ respectively. For each observation night, the atmospheric extinction coefficient of the B filter was the most and that of the I filter was the least, proving the inverse relationship between wavelength of incident radiation and the atmospheric extinction coefficient. These studies signify that generally, holding other factors constant, an electromagnetic wave of greater wavelength would get attenuated less and would thus have greater intensity post traversing the atmosphere.

\section{Seasonal Variations}

There're seasonal variations that affect the magnitude of extinction. Generally, at a given site, the magnitude of attenuation of incident light is greater in summers when the atmosphere is more humid than in winter when the atmosphere is relatively dry. Siher, Benkhaldoun and Fossat (2003) observed the maximum atmospheric extinction values during the local summer months of June, July and August. The coefficient of extinction reached 0.138 magnitudes in summer 
and decreased to 0.102 magnitudes for light wavelength $510 \mathrm{~nm}$ in winter at the Oukaimeden site in Morocco . Hale et. al (2017) analyzed data of over 30 years for 6 international observatories. For four of six, they found the extinction coefficient to be much higher in summer than in winter. For the other two, the extinction coefficients were mostly consistent, but statistically the extinction coefficient was slightly but not significantly greater in summer than in winter. Green's (1992) study concurred with the results concluded by Siher et. al and Hale et. al as he observed a smaller magnitude of extinction coefficient in the winter, dry climate for all zenith distances and heights above sea level.

\section{Importance of Accurate Calculation of Extinction}

\section{Photometry}

Astronomical photometry is the technique used to determine the Intrinsic Luminosity (the power output measured is watts) of a stellar object. If we have the apparent brightness/ intensity of radiation (I) and the distance between the stellar object and the Earth $(\boldsymbol{d})$, Intrinsic Luminosity $(\boldsymbol{L})$ can be calculated using the Inverse Square Law:

$$
I=L /\left(4 \pi d^{2}\right)
$$

Apparent brightness, measured in $W / \mathrm{m}^{2}$, is how bright a stellar object appears above the Earth's Atmosphere (Hughes, 2006). A significant intensity of light is attenuated by the atmosphere. Thus, to accurately determine and subsequently calculate the Intrinsic Luminosity of the stellar object, the measurement of the amount of radiation attenuated is extremely important.

This calculated intrinsic Luminosity is further used by astronomers to determine stellar characteristics like size, mass and lifetimes.

\section{Size}

Stephan Boltzmann's law expresses the relationship between the intrinsic luminosity and the size of the star (Eker et. al, 2015).

$$
L=\boldsymbol{\sigma} * T^{4} * A
$$

Here, $\boldsymbol{\sigma}$ is known as the Boltzmann's constant is equal to $5.67 * 10^{-7}, \boldsymbol{A}$ equals the surface area $\left(4 * \boldsymbol{\pi} * \boldsymbol{r}^{2}\right)$ and $\boldsymbol{T}$ equals the temperature (in $\mathrm{K}$ ) of the stellar object. The color temperature can be approximated using data collected from the radiation spectra and employing Wien's Law/ Blackbody radiation law:

$$
T=\frac{b}{\lambda_{(\max )}}
$$

Here, $\boldsymbol{b}$ is known as Bien's constant and is equal to $2.898 * 10^{7} K \AA, \boldsymbol{\lambda}_{\max }$ is the peak of the blackbody spectrum. The temperature can also be calculated using the $(\boldsymbol{B}-\boldsymbol{V})$ color index using the equation:

$$
T=4600 K\left(\frac{1}{0.92(B-V)+1.7}+\frac{1}{0.92(B-V)+0.62}\right)
$$

The term $(\boldsymbol{B}-\boldsymbol{V})$ is the difference between the magnitudes observed from the B filter and the $\mathrm{V}$ filter of the photometric system. 
Wien's Law assumes that the stars are perfect blackbodies, which they are not due to the presence of absorption lines that shift the maximum wavelength. This is primarily why astronomers refrain from using Wien's Law. The color index method is more suited to calculate the temperature because it averages flux over a wavelength interval (B$\mathrm{V})$. This is why the color index method is primarily used to determine the temperature of the star in photometry (Cenadelli, Potenza, \& Zeni, 2012).

Mass

The mass- luminosity relationship was first noted by Arthur Edington, who put forth that for the main sequence stars in the main sequence in the H-R diagrams, $\boldsymbol{L} \propto \boldsymbol{M}^{\boldsymbol{\alpha}}$ where $\boldsymbol{\alpha}$ is the fitting exponent and varies with respect to the mass of the star because stars with different masses have varying internal structures and ways to produce energy. The different values of $\boldsymbol{\alpha}$ as a function of mass from varying studies is presented in Table 1:

Table 1: Variability across $\boldsymbol{\alpha}$ as a function of mass of star from different studies

\begin{tabular}{|l|r|r|}
\hline Study & Mass Range (in Mass of Sun) & $\alpha$ \\
\hline Duric (1999) & $M>0.43$ & 4 \\
\hline & $M<0.43$ & 2.3 \\
\hline Salaris \& Cassisi (2008) & $2<M<20$ & 3.6 \\
\hline & $0.5<M<2$ & 4.5 \\
\hline Griffiths et. al (1998) & $0.2<M<0.5$ & 2.6 \\
\hline & & 2.44 \\
\hline & $0.4<M<5.01$ & 4.16 \\
\hline Demircan \& Kahraman (1991) & $5.01<M$ & 3.51 \\
\hline & $0.1<M<18.1$ & 3.704 \\
\hline & $0.7>M$ & 2.616 \\
\hline & $0.7<M$ & 3.916 \\
\hline
\end{tabular}

\section{Lifetime}

The lifetime of a star is determined by its mass and luminosity since its mass tells the quantity of fuel it has while the luminosity is a measure of the star's total energy output. The lifetime of a star is thus equal to total fuel available divided by the power output and is therefore proportional to:

$$
M / L \text { or } \frac{1}{M^{\alpha-1}}
$$




\section{Conclusion}

A review of existing literature on calculating magnitude of atmospheric extinction was conducted to analyze the contribution of and quantify the 3 primary causes of attenuation (Molecular Absorption, Rayleigh Scattering, Aerosol Scattering). Aerosol scattering is sought to be the biggest influencer in variability of attenuation due to everchanging change in number of suspended particles (water droplets, dust, artificial pollution). Generalized trends as a factor of seasonal variations, wavelength of incident light, and altitude of the observatory were synthesized. The wavelength of incident light and the altitude of the observatory were found to be negatively related to the amount of extinction; On average, all studies analyzed signified a greater amount of extinction in hot and humid environments than in cold and dry, with differences varying as a factor of other constituents (change of number of suspended atmospheric particles with seasons). The reduction in attenuation due to increased altitude is due to the incident rays traversing a shorter air mass, while the increase of extinction in hot and humid weather can be attributed to an increase in Aerosol Scattering due to the influx of dusty aerosols, mineral dust, and water vapor in the atmosphere.

Additionally, the importance and implications of accurate measurements of magnitude of extinction was highlighted by signifying its importance in precisely calculating the intrinsic luminosity of stars which is further used to determine stellar characteristics (size, mass, and lifetime) in the main sequence of the Hertzsprung-Russell diagram.

\section{References}

A.T. Young and W.M. Irvine, 1967, Astron. J, 72, pp945-950.

Baker, W. B., Parthasarathy, A. B., Busch, D. R., Mesquita, R. C., Greenberg, J. H., \& Yodh, A. G. (2014). Modified Beer-Lambert law for blood flow. Biomedical optics express, 5(11), 4053-4075.

https://doi.org/10.1364/BOE.5.004053

Capua, R. D., Offi, F., \& Fontana, F. (2014). Check the Lambert-Beer-Bouguer law: A simple trick to boost the confidence of students toward both exponential laws and the discrete approach to experimental physics. European Journal of Physics, 35(4). doi:10.1088/0143-0807/35/4/045025

Cenadelli, D., Potenza, M., \& Zeni, M. (2012). Stellar temperatures by Wien's law: Not so simple. American Journal of Physics, 80(5), 391-398. doi:10.1119/1.3699958

D.W. Ballentyne and D.R. Lovett, A Dictionary of Named Effects and Laws in Chemistry, Physics, and Mathematics, 3rd Ed. Chapman and Hall Ltd., London 1970, p. 188.

Demircan, O., \& Kahraman, G. (1991). Stellar mass-luminosity and mass-radius relations. Astrophysics and Space Science, 181(2), 313-322. doi:10.1007/bf00639097

Duric, N. (1999). 1.3.8 Mass -Luminosity Relation. In N. Duric (Author), Advanced astrophysics (pp. 19-20). Lincom.

Eker, Z., Soydugan, F., Soydugan, E., Bilir, S., Gökçe, E. Y., Steer, I., . . Demircan, O. (2015). Main-Sequence Effective Temperatures From A Revised Mass-Luminosity Relation Based On Accurate Properties. The Astronomical Journal, 149(4), 131. doi:10.1088/0004-6256/149/4/131

Green, D. W. (1992). MAGNITUDE CORRECTIONS FOR ATMOSPHERIC EXTINCTION. Retrieved July 12, 2020, from http://www.icq.eps.harvard.edu/ICQExtinct.htm 
Griffith, S. C., Hicks, R. B., \& Milone, E. F. (1988). A re-examination of mass-luminosity relations from binary-star data. Journal of the Royal Astronomical Society of Canada, 82, 1-12. Retrieved July 29, 2020, from http://adsabs.harvard.edu/full/1988JRASC..82...1G

Hale, S. J., Chaplin, W. J., Davies, G. R., Elsworth, Y. P., Howe, R., Lund, M. N., . . Rhodes, E. J., Jr. (2017). Atmospheric Extinction Coefficients in the Ic Band for Several Major International Observatories: Results from the BiSON Telescopes, 1984-2016. Astronomical Journal, 154(89).

Hayes, D. S., \& Latham, D. W. (1975). A REDISCUSSION OF THE ATMOSPHERIC EXTINCTION AND THE ABSOLUTE SPECTRAL_DENSITY DISTRIBUTION OF VEGA. Retrieved July 12, 2020, from http://articles.adsabs.harvard.edu/cgi-bin/nph-iarticle query?bibcode=1975ApJ...197..593H

Hughes, D. W. (2006). The Introduction Of Absolute Magnitude. Journal of Astronomical History and Heritage, 9 , 173-179.

Jurado Vargas, M., Merchán Benítez, P., Sánchez Bajo, F., \& Astillero Vivas, A. (2002). Measurements of atmospheric extinction at a ground level observatory. Astrophysics and Space Science, 279(3), 261-269.

doi:http://dx.doi.org/10.1023/A:1015184127925

Kasten, F., \& Young, A. T. (1989). Revised optical air mass tables and approximation formula. APPLIED OPTICS, $28(22)$.

McLinden, C. (1999, July 22). Mie Scattering. Retrieved July 12, 2020, from https://www.ess.uci.edu/ cmclinden/link/xx/node19.html

Penndorf, R. (n.d.). Tables of the Refractive Index for Standard Air and the Rayleigh Scattering Coefficient for the Spectral Region between 0.2 and $20.0 \mu$ and Their Application to Atmospheric Optics. Journal of the Optical Society of America, 47(2), 176-182.

Rozenberg, G. V. (1966). Twilight: A Study in Atmospheric Optics (New York:

Plenum Press), translated from the Russian by R. B. Rodman, p. 160.

R.H. Hardie, 1962, 'Photoelectric Reductions', Chapter 8 of Astronomical Techniques, W.A. Hiltner (Ed), Stars and Stellar Systems, II (University of Chicago Press: Chicago), pp 178-208.

Salaris, M., \& Cassisi, S. (2008). 5.7 The Mass- Luminosity Relation. In Evolution of stars and stellar populations (pp. 138-140). Chichester, West Sussex: John Wiley et Sons.

Siher, E. A., Benkhaldoun, Z., \& Fossat, E. (2003). Diurnal Atmospheric Extinction Over Oukaimeden Observatory Using IRIS Database: 1989- 1997. Experimental Astronomy, 13, 159-170.

Wang, J., \& Zhong, Z. (2018). Revisiting the mass-luminosity relation with an effective temperature modifier. Astronomy \& Astrophysics, 619. doi:10.1051/0004-6361/201834109 